\title{
Wie gefährlich ist die Gabe von Sauerstoff?
}

\author{
Das Pickwick-Syndrom ist gekennzeichnet durch eine massive \\ Adipositas mit alveolärer Hypoventilation und restriktiver \\ Lungenfunktionsstörung. Wegen der Hypoventilation erhalten \\ die Patienten oft Sauerstoff, doch der Einfluss dieser Maßnahme \\ auf den $\mathrm{pH}$-Wert im Blut war in diesem Zusammenhang bisher \\ nur wenig untersucht. \\ Thorax 2014; 69: 346-353
}

Beim Pickwick-Syndrom oder ObesitasHypoventilationssyndrom (OHS) führt schon die Gabe herkömmlicher inspiratorischer Sauerstoffkonzentrationen zu einer Hypoventilation und in der Folge zu erhöhten arteriellen $\mathrm{CO}_{2}$-Partialdrücken mit begleitender respiratorischer Azidose. Zu diesem Ergebnis kommen C. A. Hollier et al. in einer randomisierten Crossoverstudie.

Untersucht wurden 14 Patienten mit stabilem OHS, definiert als Body-Mass-Index $(B M I) \geq 30 \mathrm{~kg} / \mathrm{m}^{2}$ und eine auch im Wachzustand bestehende Hyperkapnie mit einem $\mathrm{paCO}_{2}$ von $>6 \mathrm{kPa}$. Ihnen wurden 14 nach Geschlecht und Alter gematchte gesunde Kontrollpersonen mit einem BMI $<30 \mathrm{~kg} / \mathrm{m}^{2}$ und laut Spirometrie normaler Lungenfunktion gegenübergestellt. Alle Teilnehmer atmeten für 20 min über ein Kreissystem eine inspiratorische Sauerstofffraktion $\left(\mathrm{FiO}_{2}\right)$ von 0,28 oder 0,5 ein. Es folgte eine Auswaschperiode von $45 \mathrm{~min}$. Danach atmeten die Teilnehmer die jeweils andere $\mathrm{FiO}_{2}$ ein. Beurteilt wurden die Auswirkungen der beiden Sauerstoffgaben auf den $\mathrm{paCO}_{2}$ im arterialisierten venösen Blut, auf den pH-Wert, das Atemminutenvolumen (AMV) sowie den Totraumanteil (Verhältnis physiologischer Totraum zu Atemzugvolumen) und zwischen Gesunden und OHS-Patienten verglichen.

Die Auswertung zeigte bei den Patienten unter beiden Sauerstoffkonzentrationen eine Sauerstoffsättigung von 98 bis $100 \%$. Unter der $\mathrm{FiO}_{2}$ von $0,28 \mathrm{kam}$ es nach 20 min zu einer deutlichen Zunahme des $\mathrm{paCO}_{2}$ um im Mittel 0,3 kPa, bei nur minimaler Veränderung des AMV und einer
Zunahme des Totraumanteils um im Mittel $1 \%$. Bei einer $\mathrm{FiO}_{2}$ von 0,5 stieg der $\mathrm{paCO}_{2}$ weiter an (im Mittel um $0,5 \mathrm{kPa}$ ), begleitet von einer Azidose mit einem pH-Wert von im Mittel 7,346. Das AMV sank innerhalb von $5 \mathrm{~min}$ um $1,21 / \mathrm{min}$, nahm dann in unterschiedlichem Ausmaß wieder zu und erreichte bzw. übertraf den Ausgangswert bei jeweils 4 Patienten. Bei 6 Patienten blieb das AMV unterhalb des Ausgangswerts. Diese Verminderung des AMV war bei gleichbleibender Atemfrequenz auf ein verringertes Atemzugvolumen zurückzuführen. Der Totraumanteil stieg um 3\%. Es fand sich eine negative Korrelation zwischen der Veränderung des $\mathrm{paCO}_{2}$ und der des $\operatorname{AMV}(\mathrm{r}:-0,60)$. In der Kontrollgruppe veränderte keine der beiden Sauerstoffkonzentrationen den $\mathrm{paCO}_{2}$ oder $\mathrm{pH}$-Wert; unter der $\mathrm{FiO}_{2}$ von 0,5 kam es allerdings bei einer leicht erhöhten Atemfrequenz zu einem Anstieg des AMV und des Totraumanteils (um 15 bzw. 4\%).

\section{Fazit}

Die Autoren kommen zu dem Schluss, dass eine zusätzliche Sauerstoffgabe in durchaus üblichen Dosierungen bei Patienten mit Pickwick-Syndrom zu einer klinisch relevanten Hypoventilation führen kann, die sich auf den pH-Wert auswirkt. Bei instabilen Patienten könnte diese Veränderung sogar noch stärker ausfallen. Dementsprechend sollte die Sauerstofftherapie bei Pickwick-Syndrom nur unter Überwachung der Sauerstoffsättigung erfolgen, mit Zielwerten zwischen 88 und $92 \%$. Regelmäßige Blutgasanalysen seien empfehlenswert.

\section{Dr. Elke Ruchalla, Trossingen}

Ausschreibung

\section{Forschungspreis der René-Baumgart- Stiftung 2015}

Die gemeinnützige René-Baumgart-Stiftung zur Förderung der medizinischen Forschung im Krankheitsbereich des Lungenhochdrucks bei Kindern und Erwachsenen schreibt den Forschungspreis 2015 für wissenschaftliche Arbeiten auf dem Gebiet der pulmonalen Hypertonie aus. Angesprochen werden Forscher, die über Lungenhochdruck arbeiten wie Internisten, Kardiologen, Pneumologen, pädiatrische Kardiologen, Herz- und Thoraxchirurgen sowie Grundlagenwissenschaftler.

Eingereicht werden können wissenschaftliche Arbeiten, die innerhalb der letzten 12 Monate vor Einreichung oder noch nicht veröffentlicht worden sind und sich klinisch oder experimentell mit der pulmonalen Hypertonie beschäftigen. Wichtige Bewertungskriterien sind Originalität, Innovation und klinische Relevanz. Die Verbesserung der Versorgung und Betreuung von Patienten steht im Vordergrund. Die Arbeit muss schwerpunktmäßig im deutschsprachigen Raum durchgeführt worden sein. Das Preisgeld ist auf $5000 €$ zur persönlichen Verfügung des Preisträgers festgesetzt. Die Verleihung erfolgt im Rahmen des 56. Kongresses der Deutschen Gesellschaft für Pneumologie und Beatmungsmedizin e.V. vom 18. bis 21. März 2015 in Berlin.

Es wird gebeten, ein Originalmanuskript bzw. einen Sonderdruck in 4-facher Ausführung, einen Lebenslauf mit Foto sowie eine deutschsprachige Zusammenfassung bis zum 31. Dezember 2014 (Posteingang) bei der René Baumgart-Stiftung, Prof. Marius M. Hoeper, Stellvertretender Vorsitzender, Rheinaustr. 94 in 76287 Rheinstetten, einzureichen. Auch eine Bewerbung via E-Mail mit PDF-Dateien und einem Bild im JPEG-Format an info@ rene-baumgart-stiftung.de ist zulässig. Der Erstautor der Arbeit gilt als Bewerber für den Preis.

Nach einer Mitteilung der René-Baumgart-Stiftung, Rheinstetten 